\title{
Alfvén Waves in Dusty Interstellar Clouds
}

\author{
N. F. Cramer and S. V. Vladimirov \\ Department of Theoretical Physics and Research Centre for Theoretical Astrophysics, \\ School of Physics, University of Sydney, NSW 2006, Australia \\ cramer@physics.usyd.edu.au
}

Received 1996 September 16, accepted 1997 February 19

\begin{abstract}
Dust particles in a plasma can be highly charged, and can carry a proportion of the negative charge of the plasma. Even if this proportion is quite small, as in interstellar dusty clouds, it can have a large effect on hydromagnetic Alfvén waves propagating at frequencies well below the ion-cyclotron frequency. In particular, the right-hand circularly polarised mode experiences a cutoff due to the presence of the dust. We generalise previous work on Alfvén waves in dusty interstellar plasmas by considering the general dispersion relation for waves propagating at an arbitrary angle with respect to the magnetic field. Wave energy propagating at oblique angles to the magnetic field in an increasing density gradient can be very efficiently damped by the Alfvén resonance absorption process in a dusty plasma, and we consider this damping mechanism for waves in interstellar clouds.
\end{abstract}

Keywords: plasmas - ISM: clouds — dust - MHD waves

\section{Introduction}

The physics of dusty plasmas has recently been studied intensively because of its importance for a number of applications in space and laboratory plasmas (Goertz 1989; Mendis \& Rosenberg 1994). Dusty plasmas are characterised as low-temperature ionised gases whose constituents are electrons, ions and micron-sized dust particulates. The latter are usually negatively charged due to the attachment of the background plasma electrons on the surface of the dust grains via collisions. The presence of dust particles (grains) changes the plasma parameters and affects the collective processes in such plasma systems. In particular, the charged dust grains can effectively collect electrons and ions from the background plasma. Thus, in the state of equilibrium the electron and ion densities are determined by the neutrality condition which is given by

$$
-e n_{e}+e n_{i}-Z_{d} e n_{d}=0,
$$

where $n_{e, i, d}$ are the concentrations of plasma electrons (with the charge $-e$ ), ions (for simplicity, we consider singly charged ions), and dust particles, respectively; the charge of the dust particle $-Z_{d}$ e can vary significantly depending on plasma parameters. Dust can be a major component of interstellar molecular clouds, and the effects of the dust on small-amplitude magnetohydrodynamic waves (Pilipp et al. 1987) and on shock waves (Pilipp \& Hartquist 1994) in the clouds have been investigated previously. For interstellar molecular clouds this charge is negative (i.e. $Z_{d}>0$ ). For $\mathrm{H}$ II regions there can be several hundred electrons per grain, while for $\mathrm{H}_{\mathrm{I}}$ regions there are only a few per grain (Spitzer 1978).

In this paper we consider the effect of the charged dust on the propagation of low-frequency hydromagnetic waves in a magnetised interstellar cloud. It is important to know the speeds and damping rates of the hydromagnetic waves in interstellar clouds because the waves transport angular momentum during cloud collapse to form protostars. There is some possible direct evidence of these hydromagnetic waves in such clouds, since they have been postulated to be the cause of the observed large widths of $\mathrm{CO}$ emission lines from molecular clouds (Arons \& Max 1975). Recently Balsara (1996) has investigated the damping of hydromagnetic waves in a partially ionised but dust-free self-gravitating plasma. It is of interest to know the effect of dust in the clouds on the speeds and damping rates of the waves. Alfvén wave propagation parallel to the magnetic field in a dusty interstellar cloud was first investigated by Pilipp et al. (1987), and was applied to the problem of wave propagation in the dustless and dusty regions of stellar outflows such as $\alpha$ Ori (Havnes, Hartquist \& Pilipp 1989). It was argued that waves which propagate with no losses out to the zone of dust formation are dissipated rapidly in the dusty region. More recently, Rao (1993) and Shukla \& Rahman (1996) focused on the very low frequency waves that are affected by the motion of the dust grains themselves and by non-zero pressures, while Mendis \& Rosenberg (1992) and Shukla (1992) considered the circularly polarised electromagnetic 
waves propagating parallel to the magnetic field in a plasma with static dust grains, in particular the case of frequency much less than the ion-cyclotron frequency. Birk, Kopp \& Shukla (1996) have derived generalised magnetohydrodynamic equations for partially ionised dusty plasmas including thermal pressure gradient terms, and have used them to investigate low-frequency Alfvén-like modes and tearing instabilities, in particular for dusty plasmas with a large proportion of the negative charge on the dust grains. The effects of dust on the propagation of shock waves in interstellar clouds, including the rotation of the magnetic field in an obliquely propagating shock, have been considered by Pilipp \& Hartquist (1994).

Even if the proportion of negative charge on the dust grains compared to that carried by free electrons is quite small (typically $\approx 10^{-4}$ in interstellar clouds), it can have a large effect on hydromagnetic Alfvén waves propagating at frequencies well below the ioncyclotron frequency, as was shown by Pilipp et al. (1987). In particular, the right-hand circularly polarised mode experiences a cutoff due to the presence of the dust. In this paper we generalise the previous work by considering the general dispersion relation for waves in a magnetised plasma with dust grains, propagating at an arbitrary angle with respect to the magnetic field. The wave frequencies are supposed to be much less than the ion-cyclotron frequency. The character of the waves at frequencies less than the ion-cyclotron frequency is radically altered when a proportion of the negative charge resides on the dust grains, in analogy with the case of electromagnetic waves in plasmas in solids with unequal electron and hole numbers (Baynham \& Boardman 1971). With a negligibly small charge on the dust grains, the waves have the usual shear and compressional Alfvén wave properties, while for a non-zero charge on the grains the waves are better described as circularly polarised whistler or helicon waves extending to low frequencies (Mendis \& Rosenberg 1994).

Waves that propagate obliquely to the magnetic field in a nonuniform plasma can encounter the Alfvén resonance, where the wavenumber perpendicular to the magnetic field direction becomes infinite (in the limit of zero electron temperature) and wave energy can be absorbed. This process is believed to be responsible for the heating of the solar corona (Ionson 1978), and has been used as an auxiliary heating mechanism for laboratory plasmas (Hasegawa \& Chen 1976). Alfvén resonance absorption of waves in dusty interstellar molecular clouds could play a role in their energy balance and in the magnetic braking of protostellar clouds. We show here that the behaviour of the waves near the Alfvén resonance is strongly modified by the presence of dust.
The paper is organised as follows. In Section 2 the general dispersion relation for Alfvén waves propagating at arbitrary angles with respect to the external magnetic field is derived. In Section 3, we present results of the dispersion relation for various situations and demonstrate the modification of the Alfvén resonance absorption in the presence of dust. In Section 4 we briefly discuss the results obtained.

\section{Model and Wave Equations}

As in Pilipp et al. (1987) we consider small-amplitude waves in a static uniform molecular cloud consisting of neutral atomic and molecular species, the ionised atomic and molecular species, the electrons, neutral dust grains, and negatively charged dust grains. The cloud is assumed to be cold, so that the gas pressures of all the species may be neglected. A four-fluid model of the plasma is used, which employs the linearised fluid momentum equations for plasma ions (singly charged), electrons, neutral molecules and charged dust grains [we neglect the motion of neutral dust grains that was included by Pilipp et al. (1987)]:

$$
\begin{aligned}
\frac{\partial \mathbf{v}_{i}}{\partial t}= & \frac{e}{m_{i}}\left(\mathbf{E}+\mathbf{v}_{i} \times \mathbf{B}_{0}\right)-\nu_{i n}\left(\mathbf{v}_{i}-\mathbf{v}_{n}\right) \\
& -\nu_{i d}\left(\mathbf{v}_{i}-\mathbf{v}_{d}\right), \\
\frac{\partial \mathbf{v}_{n}}{\partial t}= & -\nu_{n i}\left(\mathbf{v}_{n}-\mathbf{v}_{i}\right)-\nu_{n d}\left(\mathbf{v}_{n}-\mathbf{v}_{d}\right), \\
\frac{\partial \mathbf{v}_{d}}{\partial t}= & \frac{-Z_{d} e}{m_{d}}\left(\mathbf{E}+\mathbf{v}_{d} \times \mathbf{B}_{0}\right)-\nu_{d n}\left(\mathbf{v}_{d}-\mathbf{v}_{n}\right) \\
& -\nu_{d i}\left(\mathbf{v}_{d}-\mathbf{v}_{i}\right), \\
0= & \frac{-e}{m_{e}}\left(\mathbf{E}+\mathbf{v}_{e} \times \mathbf{B}_{0}\right)-\nu_{e n}\left(\mathbf{v}_{e}-\mathbf{v}_{n}\right) \\
& -\nu_{e d}\left(\mathbf{v}_{e}-\mathbf{v}_{d}\right),
\end{aligned}
$$

where $\mathbf{E}$ is the wave electric field, $m_{s}$ is the species mass and $\mathbf{v}_{s}$ is the species velocity in the wave. Further, $\nu_{s t}$ is the collision frequency of a particle of species $s$ with the particles of species $t$. We have neglected electron inertia and momentum exchange between ions and electrons, but have included ion and neutral molecule inertia terms because we are mainly interested in the frequency regime above the dust cyclotron frequency, where the ion and neutral molecule dynamics are important.

To complete the system of equations, Maxwell's equations (ignoring the displacement current) are used, with the conduction current density given by

$$
\mathbf{j}=e\left(n_{i 0} \mathbf{v}_{i}-n_{e 0} \mathbf{v}_{e}-n_{d 0} Z_{d} \mathbf{v}_{d}\right)
$$

where equilibrium charge neutrality is expressed by (1).

The background magnetic field $\mathbf{B}_{0}$ is assumed to be in the $z$-direction, and the steady electron, ion and 
dust densities are $n_{e 0}, n_{i 0}$ and $n_{d 0}$. The parameter $\delta=n_{e 0} / n_{i 0}$ measures the charge imbalance in the plasma, with the remainder of the charge residing on the dust particles, so that the total system is charge neutral. The equations are linearised, so that since we can define the transverse direction of wave field variation to be the $x$-axis, without loss of generality the wave fields are assumed to vary as $\exp \left(i k_{x} x+i k_{z} z-i \omega t\right)$. Thus our analysis differs from that of Pilipp et al. (1987) in that oblique propagation (i.e. non-zero $k_{x}$ ) is allowed for. We assume for simplicity that the charge on the dust particles is not affected by the wave, i.e. we neglect the dust charging effects discussed by Vladimirov (1994a,b).

Define the Alfvén speed $v_{A}=\sqrt{B_{0}^{2} / \mu_{0} \rho_{i}}$, where $\rho_{i}=m_{i} n_{i 0}$, the ion-cyclotron frequency $\Omega_{i}=$ $B_{0} e / m_{i}$ and the dust-cyclotron frequency $\Omega_{d}=$ $B_{0} e / m_{d}$. Eliminating $\mathbf{E}$ and $\mathbf{v}_{e}$, and using the assumed time dependence, we obtain, for $\omega \ll \Omega_{i}$,

$$
\begin{aligned}
-i \omega \mathbf{v}_{i}= & -\Omega_{m}\left(\mathbf{v}_{i}-\mathbf{v}_{d}\right) \times \hat{\mathbf{b}}+\frac{v_{A}^{2}}{\delta}(\nabla \times \mathbf{b}) \times \hat{\mathbf{b}} \\
& -\nu_{i n}\left(\mathbf{v}_{i}-\mathbf{v}_{n}\right)-\nu_{i d}\left(\mathbf{v}_{i}-\mathbf{v}_{d}\right),
\end{aligned}
$$

$$
\begin{aligned}
-i \omega \mathbf{v}_{n}= & \nu_{n i}\left(\mathbf{v}_{i}-\mathbf{v}_{n}\right)+\nu_{n d}\left(\mathbf{v}_{d}-\mathbf{v}_{n}\right) \\
-i \omega \mathbf{v}_{d}= & \frac{\Omega_{d}}{\delta}\left(\mathbf{v}_{i}-\mathbf{v}_{d}\right) \times \hat{\mathbf{b}}+\frac{\Omega_{d}}{\Omega_{i}} \frac{v_{A}^{2}}{\delta}(\nabla \times \mathbf{b}) \times \hat{\mathbf{b}} \\
& -\nu_{d n}\left(\mathbf{v}_{d}-\mathbf{v}_{n}\right)+\nu_{d i}\left(\mathbf{v}_{i}-\mathbf{v}_{d}\right) \\
-i \omega \mathbf{b}= & \nabla \times\left[\frac{1}{\delta}\left(\mathbf{v}_{i}-(1-\delta) \mathbf{v}_{d}\right) \times \hat{\mathbf{b}}\right]
\end{aligned}
$$

where $\mathbf{b}$ is the wave magnetic field, $\hat{\mathbf{b}}=\mathbf{B}_{0} / B_{0}$ and $\Omega_{m}=\Omega_{i}(1-\delta) / \delta$. The neglect of gas pressure in all species implies that $v_{i z}=v_{d z}=v_{n z}=0$.

\section{The Dispersion Relation}

We now write the fields in terms of circularly polarised mode amplitudes:

$$
b_{ \pm}=b_{x} \pm i b_{y}, \quad v_{i \pm}=v_{i x} \pm i v_{i y}, \quad \text { etc. }
$$

where the $+(-)$ sign corresponds to the left(right)hand circularly polarised wave.
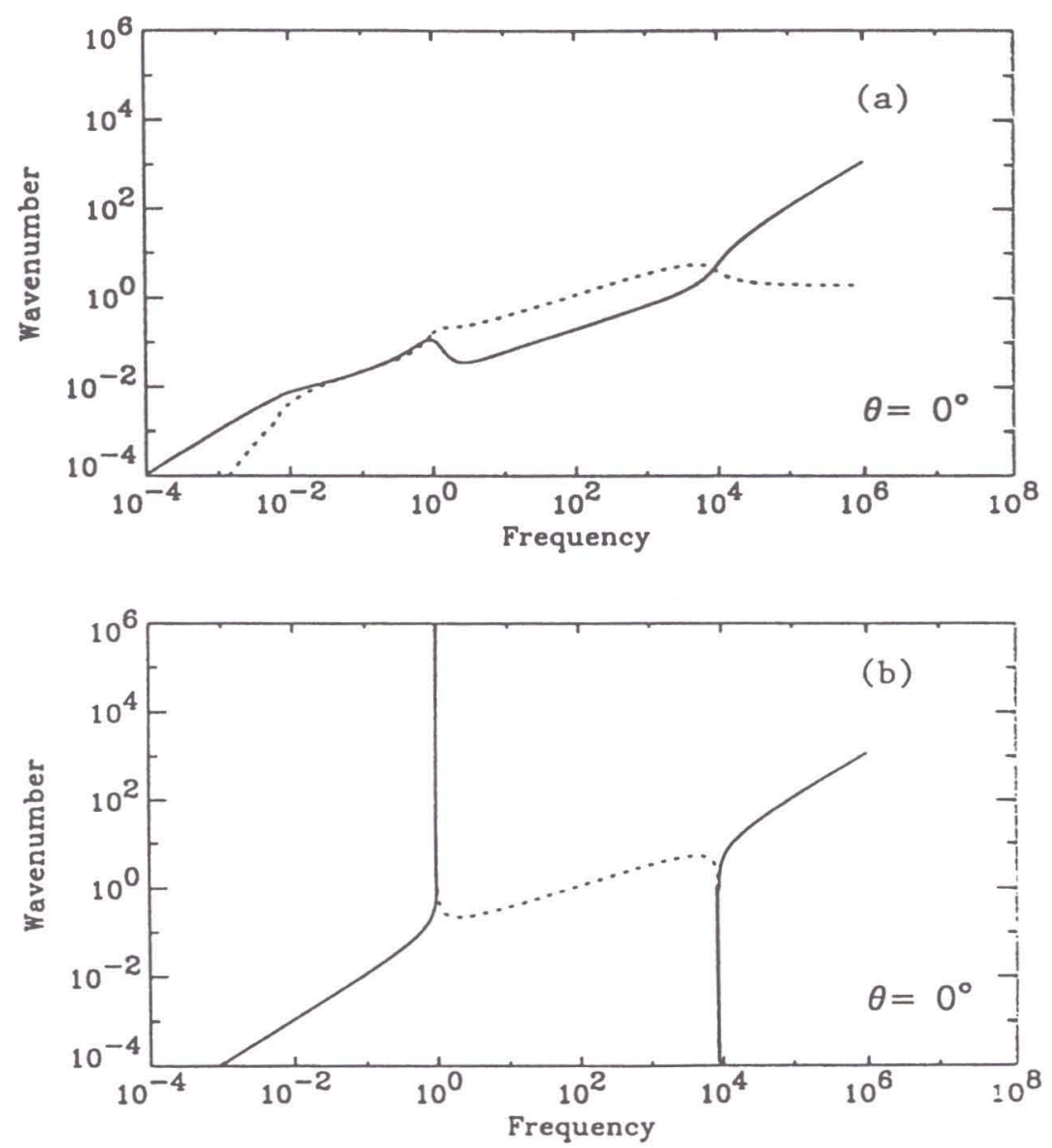

Figure 1-Wavenumber $k$ plotted against frequency $\omega$ for propagation parallel to the magnetic field, for the right-hand polarised mode. The frequency is normalised to $\Omega_{d}$, and the wavenumber to $\Omega_{d} / v_{A T}$. The dust parameter is $\delta=1-0.8 \times 10^{-4}$. (a) Collisions between species are included. (b) Collisions are neglected. 
Using (11) in (7)-(10) yields, after some algebra,

$$
\begin{gathered}
b_{ \pm}=-\frac{k_{z}}{\delta \omega} C_{ \pm} v_{i \pm} \\
{\left[D_{ \pm}-\left(k_{z}^{2}+\frac{k_{x}^{2}}{2}\right)\right] b_{ \pm}=\frac{k_{x}^{2}}{2} b_{\mp} .}
\end{gathered}
$$

Here

$$
\begin{aligned}
& C_{ \pm}= \frac{\omega \pm \delta \Omega_{d}+i\left(\nu_{d n}+\delta \nu_{d i}\right)+\nu_{d n} \nu_{n d} / \omega_{0}}{\omega \pm \Omega_{d}+i\left(\nu_{d n}+\nu_{d i}\right)+\nu_{d n} \nu_{n d} / \omega_{0}} \\
& D_{ \pm}= \\
& \frac{\delta^{2} \omega^{2}}{v_{A}^{2}}\left[\left(1+i \frac{\nu_{i n}}{\omega_{0}}\right)\left(\omega \pm \Omega_{d}+i\left(\nu_{d n}+\nu_{d i}\right)+\frac{\nu_{d n} \nu_{n d}}{\omega_{0}}\right)\right. \\
&\left.+\left(1+i \frac{\nu_{d n}}{\omega_{0}}\right)\left( \pm \Omega_{m}+i \nu_{i d}-\frac{\nu_{i n} \nu_{n d}}{\omega_{0}}\right)\right] \\
& \quad \times\left[\omega \pm \Omega_{d}+i\left(\nu_{d n}+\nu_{d i}\right)+\frac{\nu_{d n} \nu_{n d}}{\omega_{0}}\right]^{-1}
\end{aligned}
$$

with

$$
\omega_{0}=\omega+i\left(\nu_{n i}+\nu_{n d}\right)
$$

In our case of $\delta$ very close to $1, C_{ \pm} \approx 1$.

Equation (13) shows that for oblique propagation $\left(k_{x} \neq 0\right)$, the amplitudes of opposite circular polarisation are coupled together, i.e. the modes are not purely circularly polarised. We then obtain from (13),

$$
k_{x}^{2}=\frac{\left(k_{z}^{2}-D_{+}\right)\left(k_{z}^{2}-D_{-}\right)}{\left(D_{+}+D_{-}\right) /\left(2-k_{z}^{2}\right)} .
$$

The cutoffs of $k_{x}$ (where $k_{x}=0$ ) correspond to

$$
k_{z}^{2}-D_{ \pm}=0
$$

which is the parallel propagation case treated by Pilipp et al. (1987). For no collisions, a case previously treated by Shukla (1992) and Mendis \& Rosenberg (1992), the parallel propagation dispersion relation is, from (18),

$$
k_{z}^{2}=\frac{\delta^{2} \omega^{2}}{v_{A}^{2}}\left(\frac{\omega \pm \Omega_{d} \pm \Omega_{m}}{\omega \pm \Omega_{d}}\right) \text {, }
$$
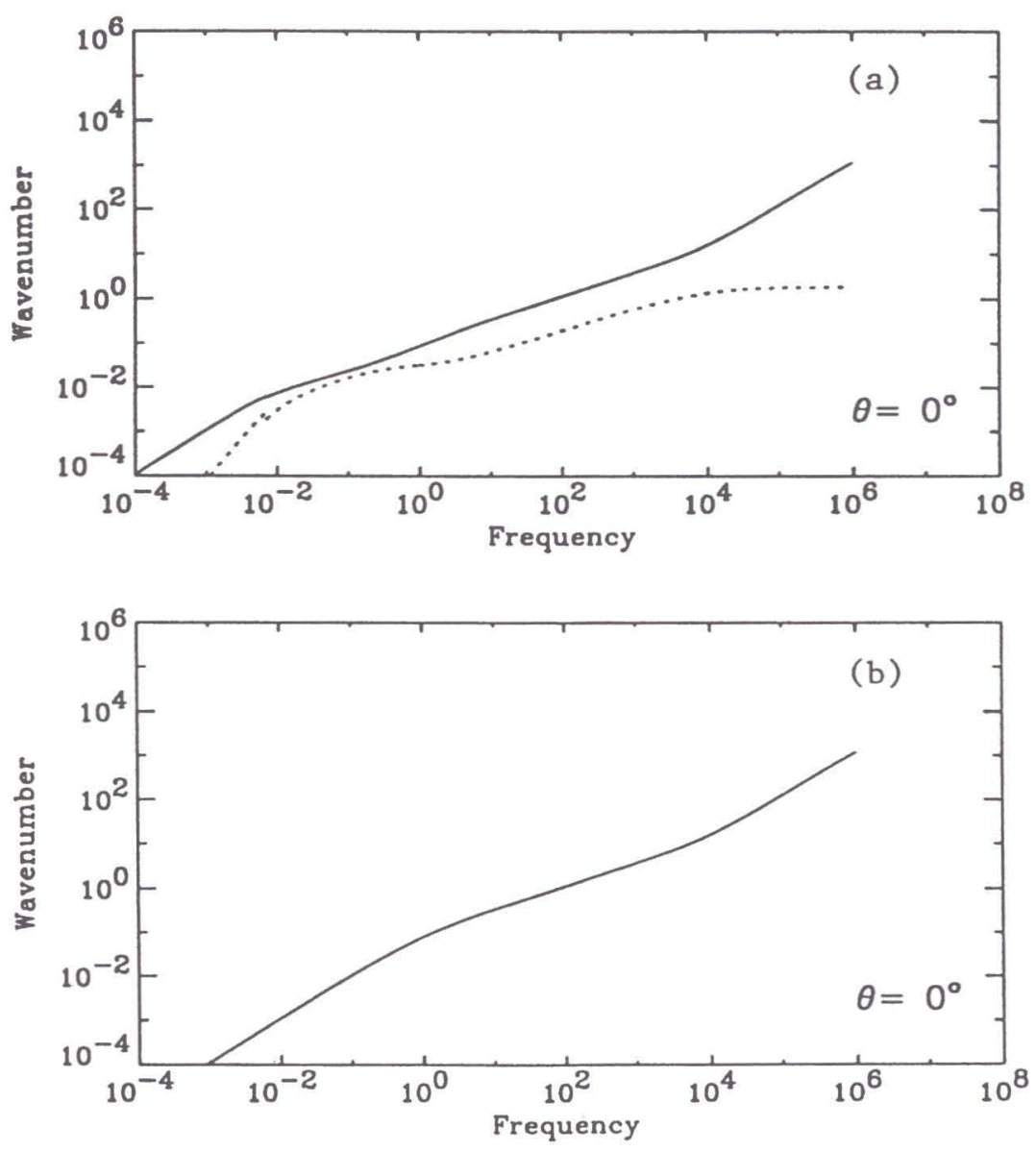

Figure 2-As for Figure 1, but for the left-hand polarised mode. 
showing, for the right-hand polarised mode, the cutoff where $k_{z}=0$ at $\omega=\Omega_{m}+\Omega_{d}$, and the resonance where $k_{z} \rightarrow \infty$ at $\omega=\Omega_{d}$. This mode was discussed by Shukla (1992). For $\Omega_{d}=0$, we obtain the modification of the parallel propagating Alfvén wave due to dust discussed by Vladimirov \& Cramer (1996) as the basis for a discussion of nonlinear effects: the right-hand circularly polarised mode with a cutoff in $k_{z}$ at $\omega=\Omega_{m}$, and the left-hand circularly polarised mode with $\omega \approx v_{A}^{2} k_{z}^{2} / \delta^{2} \Omega_{m}$ as $k_{z} \rightarrow 0$.

\section{Results}

It is instructive to use equation (18) to reproduce the result of Pilipp et al. (1987) for parallel propagation with the effects of dust and collisions, and compare it with the case where collisions between particles are neglected. As in Pilipp et al. (1987), a dense molecular cloud is considered, in which the density of molecular hydrogen is $n_{n 0}=10^{4} \mathrm{~cm}^{-3}$, $n_{i 0} / n_{n 0}=10^{-7}$, the magnetic field is $10^{-4} \mathrm{G}$ and the dominant ion species is $\mathrm{HCO}^{+}$. It is assumed that $1 \%$ of the mass is contained in spherical dust grains of radius $10^{-5} \mathrm{~cm}$ composed of material with a mass density of $1 \mathrm{~g} \mathrm{~cm}^{-3}$. We then have $1-\delta=0.8 \times 10^{-4}$. The temperature of the cloud is assumed to be $20 \mathrm{~K}$, so that the thermal pressure is approximately $10 \%$ of the magnetic pressure, and we are justified in neglecting the pressure gradient terms in equations (2)-(5). At very low frequencies and for highly electron density depleted plasmas $\left(n_{i} \gg n_{e}\right)$, dust-acoustic modes can couple to the Alfvén waves (Birk et al. 1996), but we do not consider such situations here.

We choose to plot the real part of the wavenumber (solid curve) and the imaginary part of the wavenumber (dotted curve) against the real frequency in Figures 1-6, as was done by Pilipp et al. (1987). We can thus determine the wavelength and damping length of the waves from a localised source oscillating at a given real frequency. The inverse determination of the real part of the frequency and the damping time of a wave of given real wavelength and angle of propagation is not so straightforward because the dispersion relation (17) is of eighth order in $\omega$. However, physically relevant solutions may readily be found numerically, and for lightly damped modes the ratio of imaginary to real frequency for real wavenumber is the same as the ratio of imaginary to real wavenumber for real frequency.

Figure 1a shows the plot of the real and imaginary parts of $k_{z}$ against frequency for the right-hand polarised mode. The frequency is normalised to the dust cyclotron frequency $\Omega_{d}\left(=1.12 \times 10^{-2} \mathrm{yr}^{-1}\right)$,
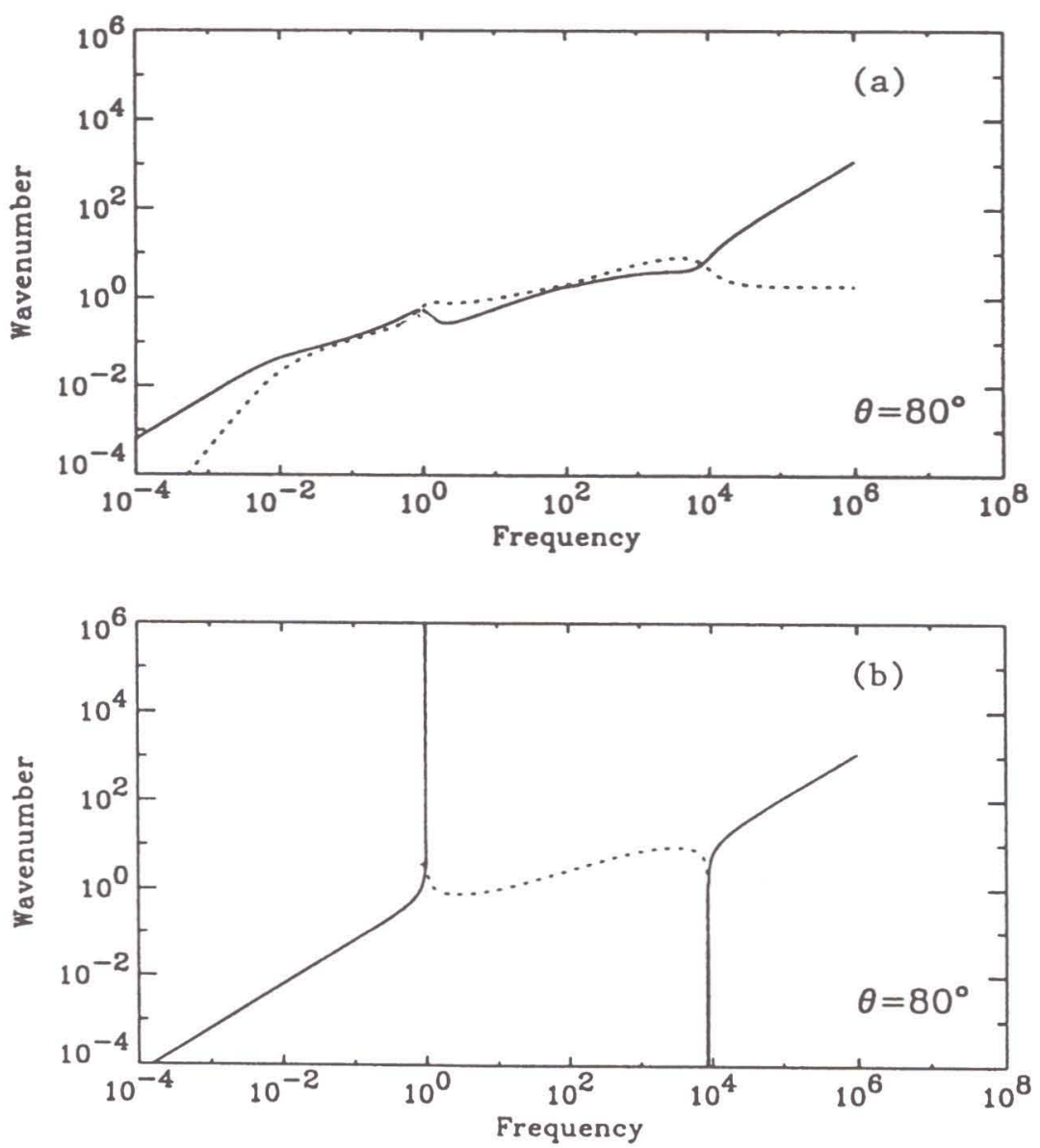

Figure 3-As for Figure 1, but for propagation at an angle of $80^{\circ}$ to the magnetic field. 

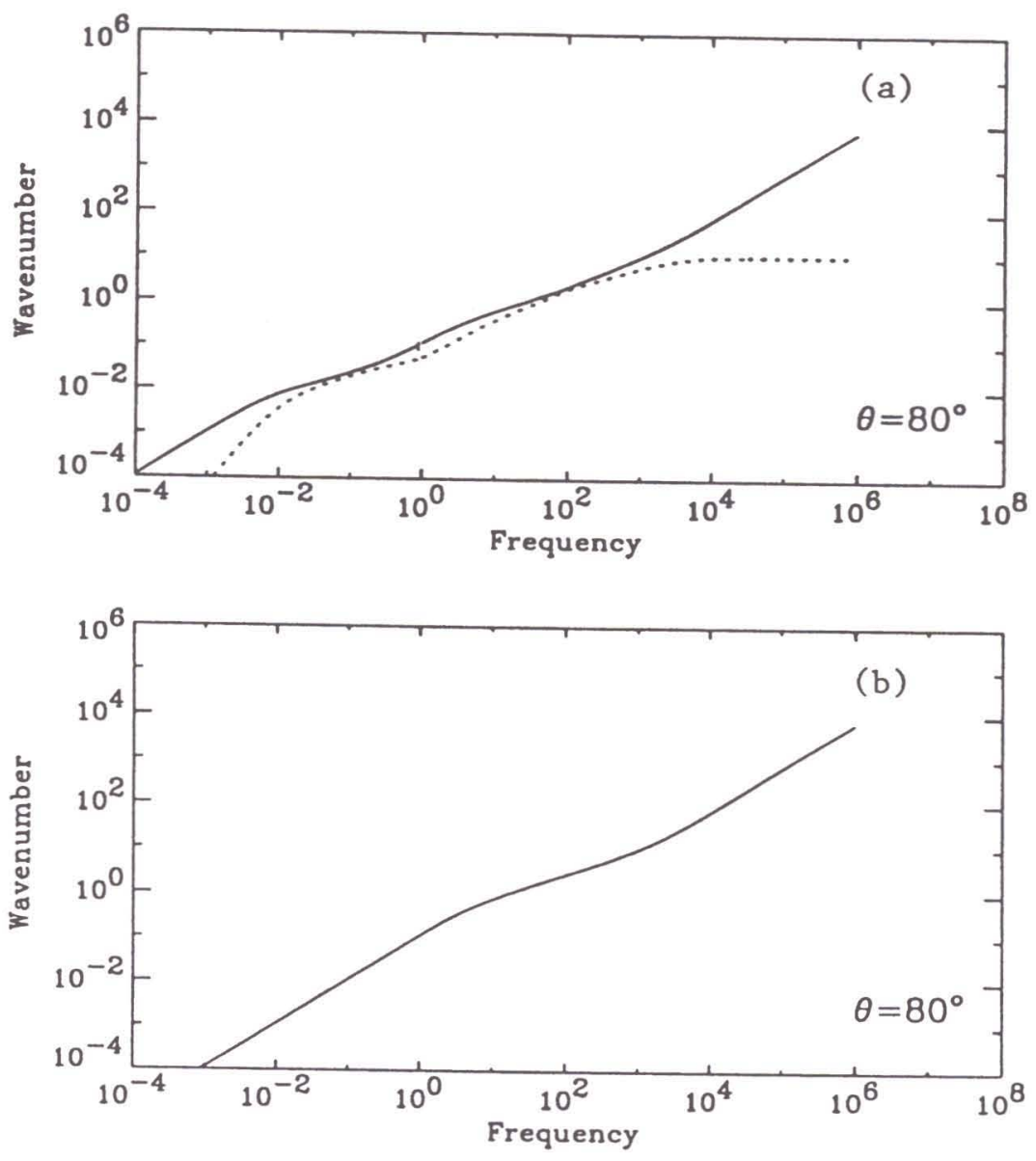

Figure 4-As for Figure 3, but for the left-hand polarised mode.

and the wavenumber is normalised to $\Omega_{d} / v_{A T}$ $\left(=7.6 \times 10^{3} \mathrm{pc}^{-1}\right)$, where $v_{A T}$ is the Alfvén speed obtained using the total mass density of the ions, neutral molecules and dust grains. We also have $\Omega_{m} / \Omega_{d}=0.88 \times 10^{4}$. The collision frequencies are the same as used in Pilipp et al. (1987). Figure 1b shows the corresponding result when all the collision frequencies are set to zero. The wave is now either purely propagating or purely evanescent, with the cutoff and resonance obtained from equation (19) evident. With collisions included (Figure 1a), the wave is heavily damped between the resonance and cutoff frequencies. Figure 2 shows the corresponding dispersion relations for the left-hand polarised mode. This mode does not experience the dust-induced resonance and cutoff behaviour.

We turn now to the oblique propagation case, for propagation with the wavenumber $k$ at an angle $\theta$ to the magnetic field, with $k_{x}=k \sin \theta$ and $k_{z}=k \cos \theta$. We have from equation (17) the following quadratic equation in $k$ describing the two independent elliptically polarised modes:

$$
\begin{aligned}
k^{4} \cos ^{2} \theta-k^{2} \frac{\left(1+\cos ^{2} \theta\right)}{2}\left(D_{+}\right. & \left.+D_{-}\right) \\
+D_{+} D_{-} & =0 .
\end{aligned}
$$

Figure 3 shows the real and imaginary parts of $k$ obtained from equation (20), plotted against frequency for $\theta=80^{\circ}$, for the right-hand polarised mode. With collisions included (Figure 3a), the real and imaginary parts of $k$ are comparable in size for frequencies between the resonance and cutoff, i.e. the relative damping of the wave is less than for the parallel propagating wave. The left-hand polarised mode at $\theta=80^{\circ}$ (Figure 4) does not show much difference to the parallel propagating case.

If $k_{z}$ is fixed and there are no collisions, there is a resonance frequency $\omega_{r}$ at which $k_{x} \rightarrow \infty$, given, from equation (17), by the condition

$$
k_{z}^{2}=\left(D_{+}+D_{-}\right) / 2 \text {. }
$$

For no collisions and small $\Omega_{d}$ this frequency is given to first order in $\Omega_{d}$ by

$$
\omega_{r}=\frac{v_{A} k_{z}}{\delta}+\frac{\delta \Omega_{d} \Omega_{m}}{2 v_{A} k_{z}}
$$

which is the generalisation to the dusty plasma of the well-known Alfvén resonance in dust-free plasmas (e.g. Hasegawa \& Chen 1976). There are also two cutoffs in $k_{x}$ determined by the vanishing of the two factors in the numerator of equation 

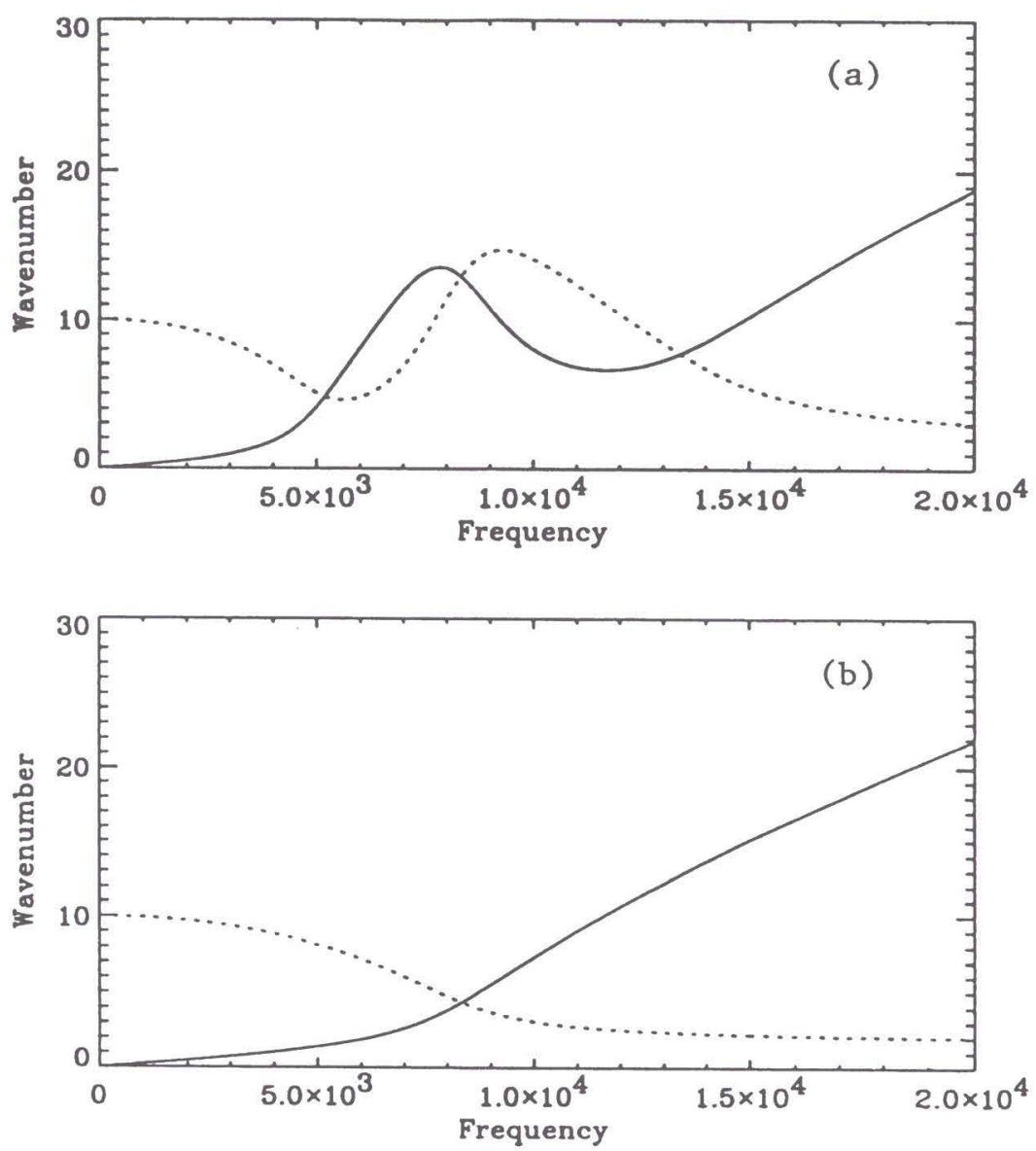

Figure 5-Wavenumber $k_{x}$ perpendicular to the magnetic field plotted against frequency $\omega$ for fixed wavenumber parallel to the magnetic field $k_{z}=10$, where the same normalisations as in Figure 1 are used. (a) Dust is present $\left(\delta=1-0.8 \times 10^{-4}\right)$ and collisions between species are included. (b) Dust is absent $(\delta=1)$ and collisions are included.

(17). In the collision-free case, and for small $\Omega_{d}$ and $\Omega_{m}$, the cutoffs occur at $\omega \approx \omega_{r} \pm \Omega_{m} / 2$, i.e. there is a cutoff-resonance cutoff triplet of frequencies (Cramer \& Vladimirov 1996 discuss the collisionless case further).

If collisions are included, there is no longer a pure resonance in $k_{x}$ at $\omega=\omega_{r}$, but the real part of $k_{x}$ attains a maximum there. This is shown in Figure $5 \mathrm{a}$, which is a plot of the real and imaginary parts of $k_{x}$ against frequency for fixed $k_{z}=10$. For our parameters, $\omega_{r} / \Omega_{d}=0.83 \times 10^{4}$. For comparison, Figure $5 \mathrm{~b}$ shows the corresponding plot for the case of no dust $(\delta=1)$. Also for comparison, Figure 6 shows the corresponding plots for no collisions between the species: Figure 6a for dust present and Figure $6 \mathrm{~b}$ for no dust. In this case the cutoff-resonance-cutoff behaviour of the dispersion relation, which is absent for the dust-free plasma, is clearly shown. We see from Figure 5 that the presence of dust still radically alters the dispersion relation in the vicinity of the Alfvén resonance in the presence of collisions: the cutoff-resonance-cutoff triplet is still discernible and strong absorption occurs over a wide range of frequencies about the Alfvén resonant frequency. Another view of the
Alfvén resonance absorption mechanism is gained by considering a wave of fixed frequency and $k_{z}$ propagating into a plasma of increasing ion density $\rho_{i}$ in the $x$-direction. The wave will encounter the cutoff-resonance-cutoff triplet at successive spatial points in the density gradient, and wave energy will be absorbed at the resonance position where condition (22) is satisfied. It has been shown by Cramer \& Vladimirov (1996) that in the collisionless case the resonance absorption in such a nonuniform plasma can be considerably enhanced by the presence of the dust, because the wave may be cut off downstream of the resonance. The same will occur in the collisional case considered here, the only difference being that the resonance absorption occurs via the collisional damping processes.

\section{Conclusions}

We have shown that the presence of dust particles which acquire a proportion of the negative charge in a plasma can strongly affect the dispersion relation of obliquely propagating Alfvén waves in interstellar dusty clouds. The consequences are that the low-frequency wave has a helicon wave character (elliptically polarised) rather than an 

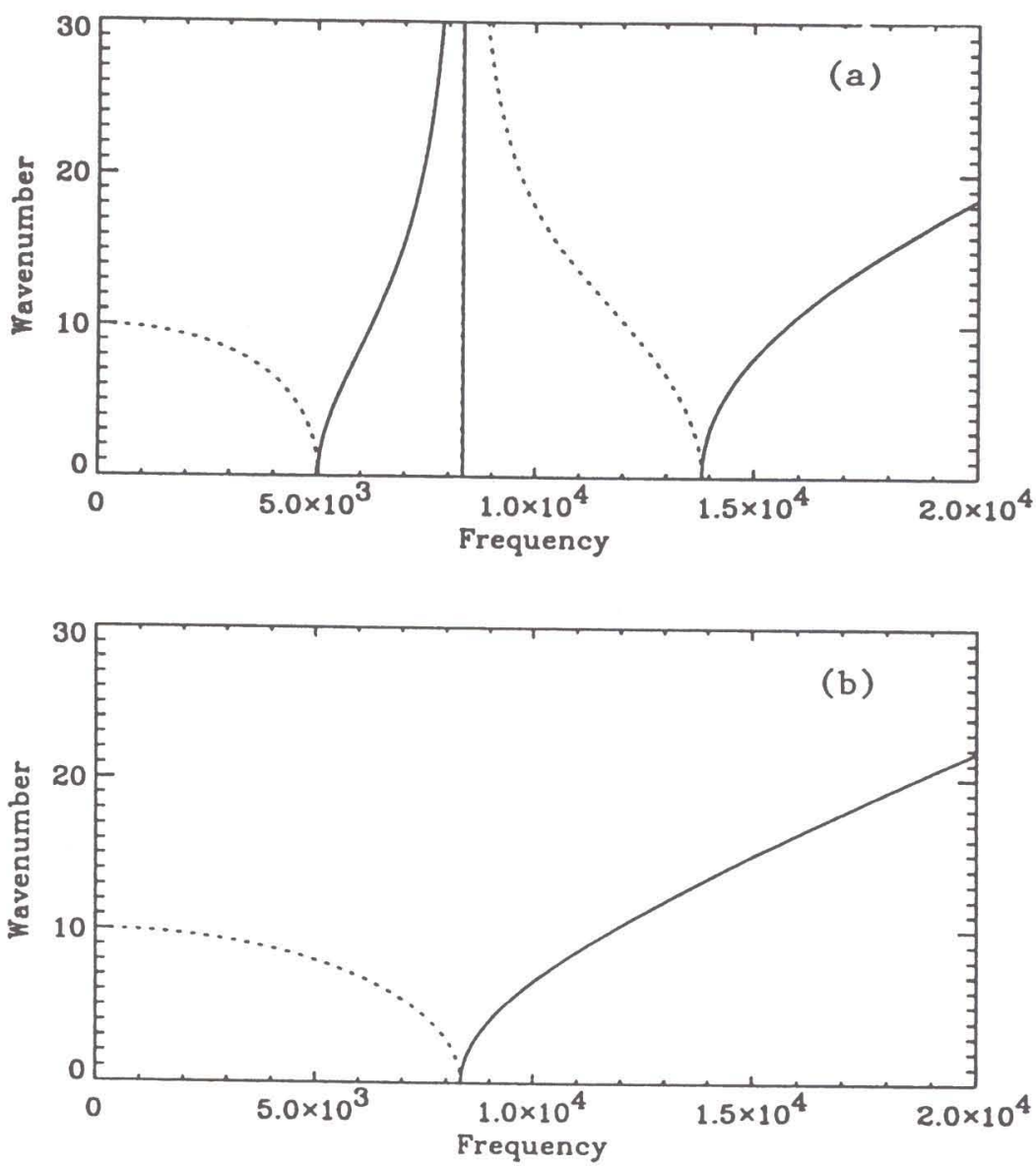

Figure 6-As for Figure 5, but collisions between species are not included.

Alfvén wave character (linearly polarised), and that the behaviour of the wave near the Alfvén resonance is modified, with possibly enhanced Alfvén resonance absorption in such a plasma. For the cloud parameters considered here, the resonant frequency is $\approx 10^{2} \mathrm{yr}^{-1}$, for a wavelength along the magnetic field of $\approx 10^{-4} \mathrm{pc}(\approx 20 \mathrm{AU})$. At that frequency, the damping length is $\approx 10$ times the wavelength for parallel propagation (and the damping time is $\approx 10^{-1}$ yr for a real wavenumber), while for oblique propagation at the resonant frequency the wavelength and damping length are both $\approx 20 \mathrm{AU}$.

We note that the dust-charge fluctuations as well as the dynamics of the dust charging process can lead to the appearance of an imaginary part in the dispersion equation (Tsytovich \& Havnes 1993), leading to a damping of the wave in addition to the collisional damping that we have considered. However, such a damping depends on the electric field component parallel to the magnetic field, which is small for Alfvén and magnetoacoustic waves in a low gas pressure plasma as considered here, so we have neglected it.

The results we have obtained in the specific context of waves in dusty interstellar clouds should also be useful for understanding the effects of propagation and absorption of Alfvén waves in multicomponent dusty plasmas such as those in planetary dust rings and the Earth's mesopause.

\section{Acknowledgments}

Support for this work has been provided by the Australian Research Council.

Arons, J., \& Max, C. E. 1975, ApJ, 196, L77

Balsara, D. S. 1996, ApJ, 465, 775

Baynham, A. C., \& Boardman, A. D. 1971, Plasma Effects in Semiconductors: Helicon and Alfvén Waves (London: Taylor and Francis)

Birk, G. T., Kopp, A., \& Shukla, P. K. 1996, Phys. Plasmas, 3,3564

Cramer, N. F., \& Vladimirov, S. V. 1996, Phys. Scripta, 53,586

Goertz, C. K. 1989, Rev. Geophys., 27, 271

Hasegawa, A., \& Chen, L. 1976, Phys. Fluids, 19, 1924

Havnes, O., Hartquist, T. W., \& Pilipp, W. 1989, A\&A, 217, L13

Ionson, J. A. 1978, ApJ, 226, 650

Mendis, D. A., \& Rosenberg, M. 1992, IEEE Trans. Plasma Sci., 20, 929

Mendis, D. A., \& Rosenberg, M. 1994, ARA\&A, 32, 419

Pilipp, W., \& Hartquist, T. W. 1994, MNRAS, 267, 801

Pilipp, W., Hartquist, T. W., Havnes, O., \& Morfill, G. E. 1987, ApJ, 314, 341 
Rao, N. N. 1993, J. Plasma Phys., 49, 375

Shukla, P. K. 1992, Phys. Scripta, 45, 504

Shukla, P. K., \& Rahman, H. U. 1996, Phys. Plasmas, 3, 430

Spitzer, L., Jr. 1978, Physical Processes in the Interstellar Medium (New York: John Wiley), p. 168
Tsytovich, V. N., \& Havnes, O. 1993, Comments Plasma Phys. Contr. Fusion, 15, 267

Vladimirov, S. V. 1994a, Phys. Plasmas, 1, 2762

Vladimirov, S. V. 1994b, Phys. Rev. E, 49, R997; ibid. 50, 1422

Vladimirov, S. V., \& Cramer, N. F. 1996, Phys. Rev. E, 54, 6762 